\title{
Adipose tissue infiltration in normal-weight subjects and its impact on metabolic function
}

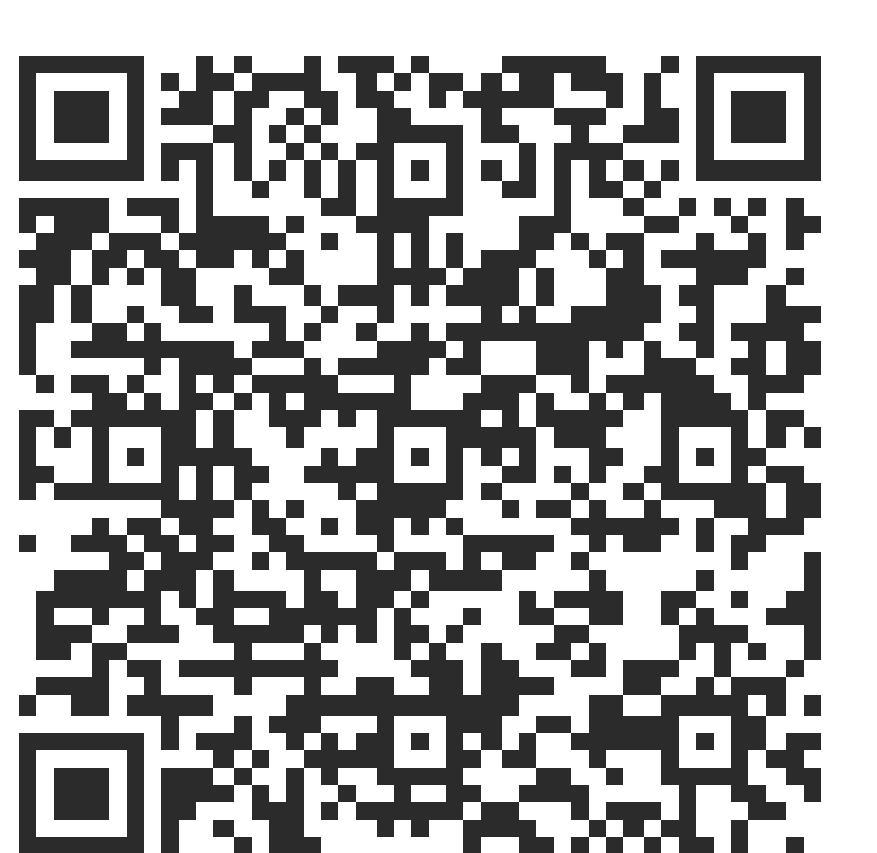

Moreno-Indias, I 1,2,3; Oliva-Olivera, W1,2,3; Castellano-Castillo, D1,2,3; Lhamyani, S²; Fernández-García, JC 1,2,3; Queipo-Ortuño MI1,2,3;

Cardona $\mathrm{F}^{1,2,3}$; Tinahones, FJ1,2,3

${ }^{1}$ Endocrinology Department. Virgen de la Victoria University Hospital. Málaga (Spain).

2Biomedical Research Laboratory. Endocrinology Department. IBIMA (Malaga).

${ }^{3}$ Spanish Biomedical Research Centre in Physiopathology of Obesity and Nutrition (CIBEROBN) Madrid, Spain

Discordant phenotypes study is crucial in the fight against obesity. Discordant phenotypes help to the understanding of obesity pathophysiology. Metabolically healthy obese individuals have paid a huge attention, whereas their lean counterparts have not received as much attention. This study deals for the first time with the differences in macrophage infiltration between metabolically healthy (MH) and unhealthy (MU) normal-weight subjects, finding a greater infiltration in subcutaneous adipose tissue of metabolically unhealthy normal-weight subjects.

Adipose tissue macrophages (ATMs) are increased in the SAT of MU
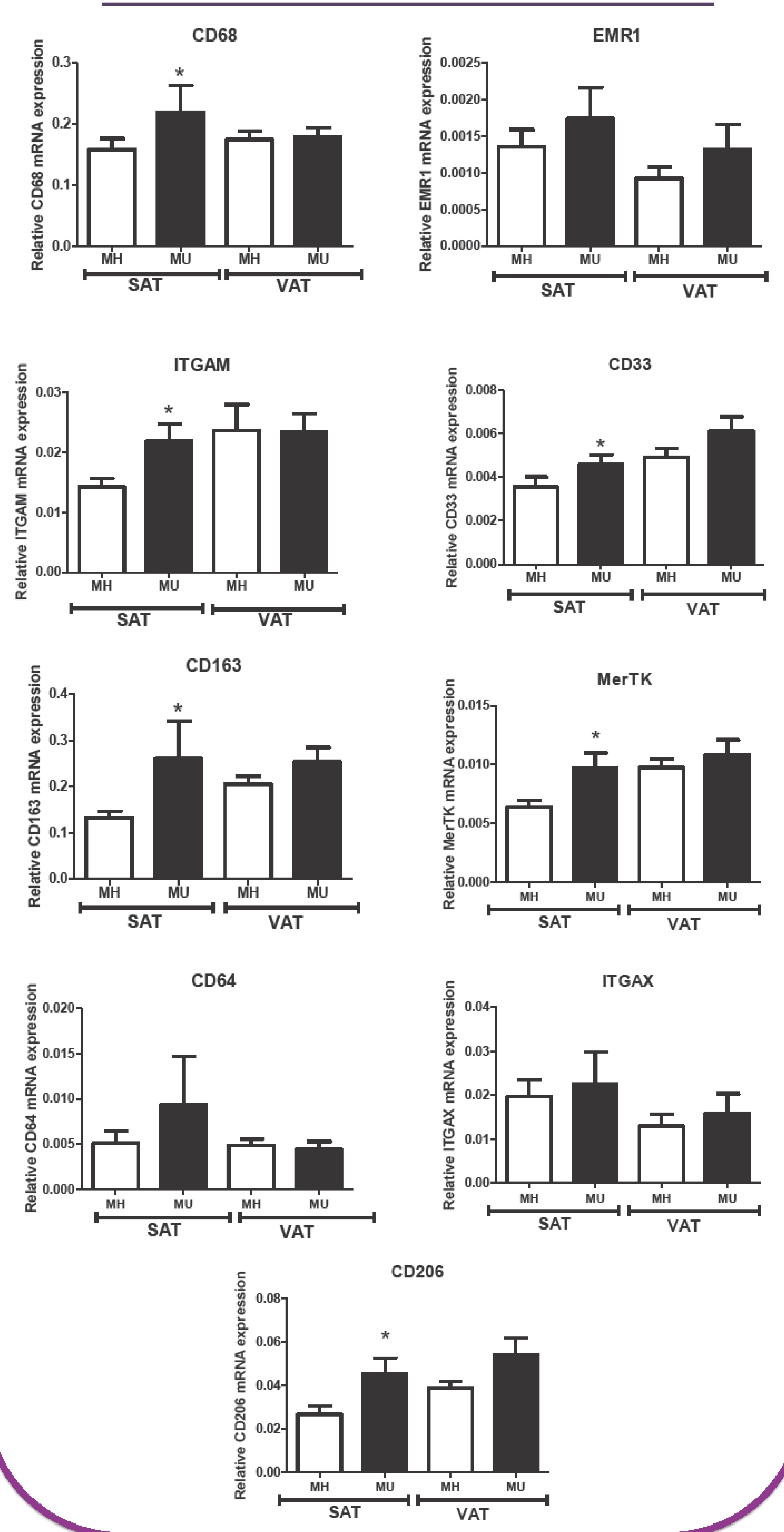

Lipogenesis and adipogenesis are decreased in the adipose tissue of MU
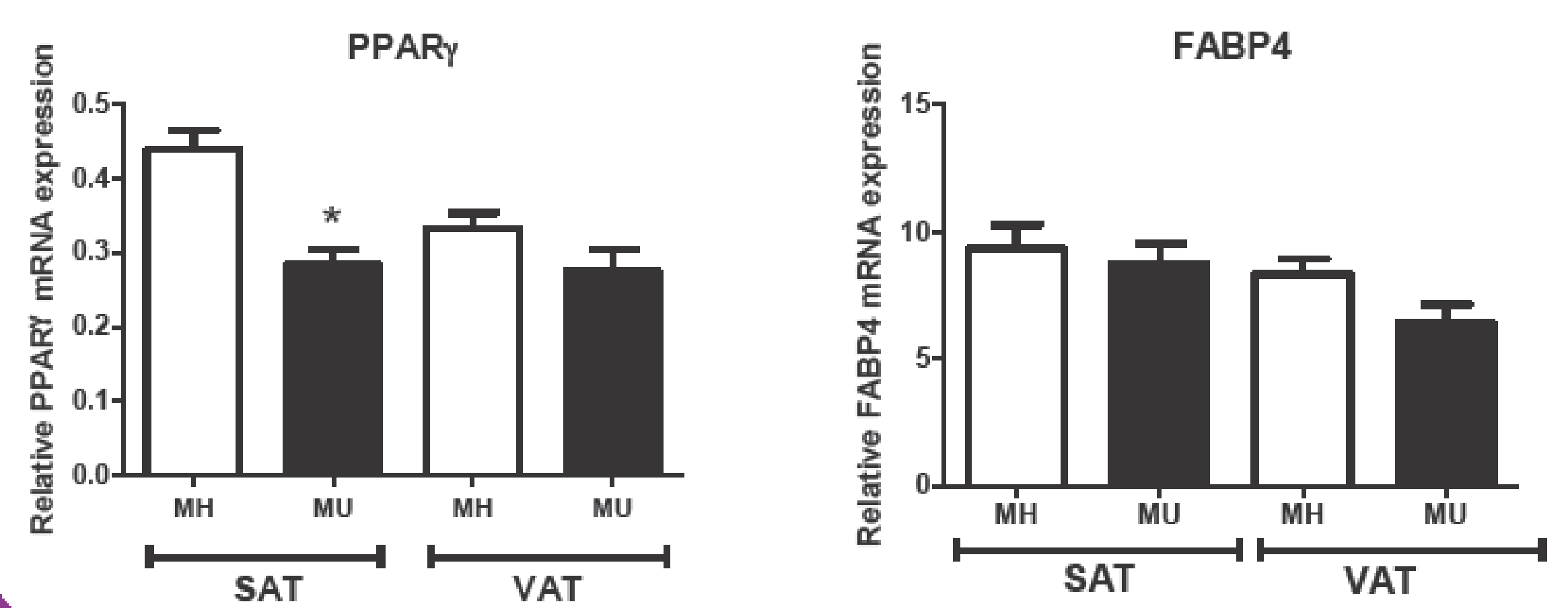

Inflammatory markers and mediators are slightly upregulated in MU adipose tissues
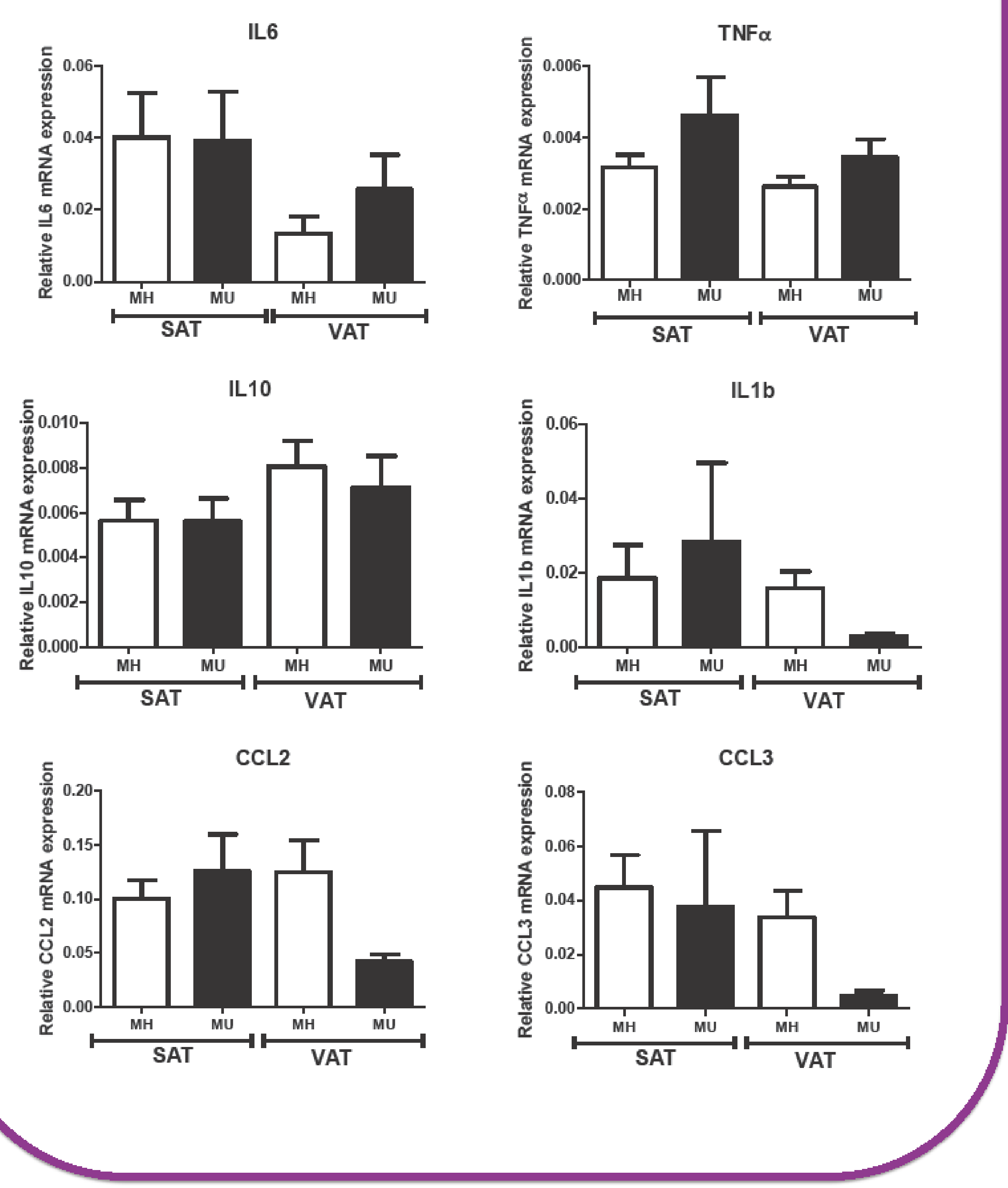

\begin{tabular}{|cccc|}
\hline Patients & MH & MU & P-value \\
\hline n (males/females) & $71(30 / 41)$ & $21(9 / 12)$ & - \\
\hline Age & $46.87 \pm 13.44$ & $53.24 \pm 15.09$ & 0.149 \\
\hline BMI (kg/m2) & $23.40 \pm 1.30$ & $24.11 \pm 1.31$ & 0.099 \\
\hline Fasting glucose (mg/dL) & $89.80 \pm 11.36$ & $101.24 \pm 10.445$ & 0.000 \\
\hline HOMA-IR score & $1.76 \pm 1.24$ & $2.60 \pm 1.29$ & 0.004 \\
\hline TG (mg/dL) & $87.85 \pm 40.66$ & $184.86 \pm 73.13$ & 0.000 \\
\hline Hip (cm) & $94.44 \pm 8.47$ & $96.76 \pm 4.38$ & 0.139 \\
\hline Waist (cm) & $82.30 \pm 7.91$ & $90.10 \pm 5.29$ & 0.000 \\
\hline Waist/Hip ratio & $0.86 \pm 0.08$ & $0.92 \pm 0.04$ & 0.068 \\
\hline Insulin ( $\mu \mathrm{Ul} / \mathrm{mL})$ & $7.89 \pm 4.98$ & $10.41 \pm 4.94$ & 0.021 \\
\hline CRP (mg/L) & $3.40 \pm 2.62$ & $9.02 \pm 22.97$ & 0.009 \\
\hline C Peptide (ng/mL) & $1.92 \pm 0.72$ & $2.38 \pm 0.558$ & 0.028 \\
\hline Cholesterol (mg/dL) & $202.87 \pm 40.39$ & $226.19 \pm 32.82$ & 0.024 \\
\hline HDL-chol (mg/dL) & $59.10 \pm 13.88$ & $45.14 \pm 8.32$ & 0.000 \\
\hline LDL-chol (mg/dL) & $124.61 \pm 32.79$ & $145.59 \pm 29.52$ & 0.015 \\
\hline SBP (mmHg) & $119.77 \pm 20.754$ & $129.14 \pm 17.48$ & 0.175 \\
\hline DBP (mmHg) & $75.20 \pm 13.154$ & $79.90 \pm 8.75$ & 0.216 \\
\hline
\end{tabular}

Differentiated cultured cells of SAT and VAT adipose tissues in MH and MU SAT from MH subjects VAT from MH subjects
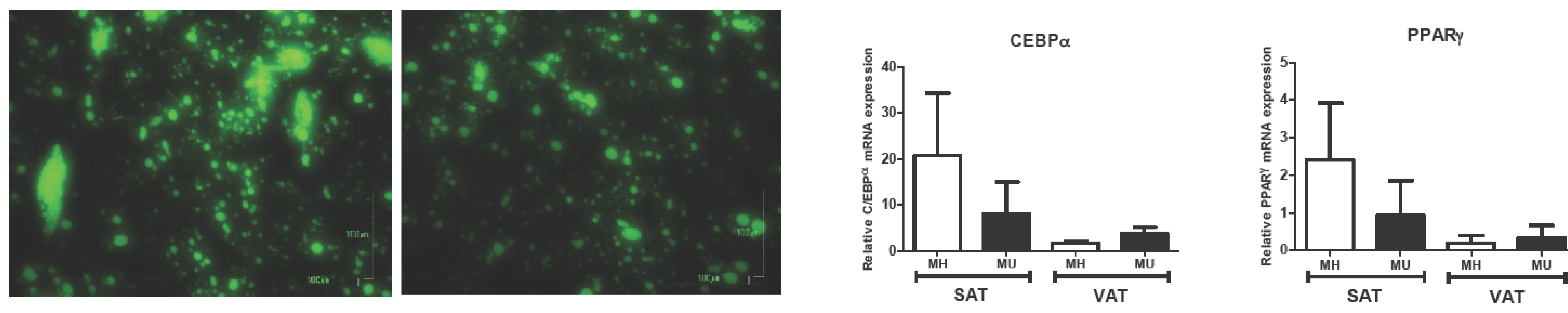

SAT from MU subjects VAT from MU subjects
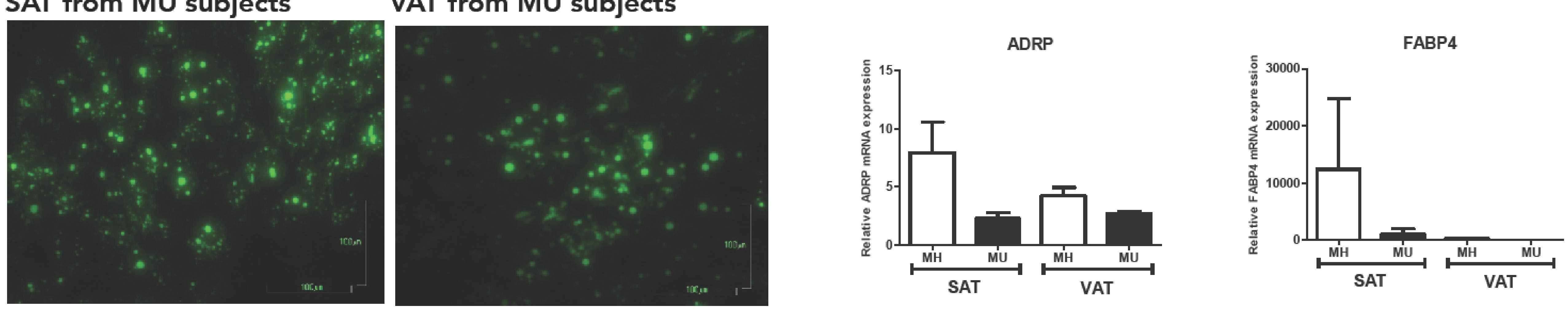

Higher adipogenic capacity of SAT from MH subjects with respect to the SAT from MU subjects.

(1) The data from this study reinforce the notion that macrophage infiltration into the SAT of MU normal-weight subjects may contribute to cause metabolic disturbances leading to diabetes or cardiovascular diseases.

- Adipogenesis in SAT seemed to be one of the complications related with the metabolic syndrome, showing a reduction in MU subjects.

- The metabolic syndrome parameter most related with macrophage/monocyte infiltration was the HDL cholesterol level.

Thus, the increase in subcutaneous ATM infiltration may explain why some normal-weight and obese individuals develop metabolic disease whereas others remain MH. Further molecular discoveries will facilitate health management strategies targeting the adipose tissue function.

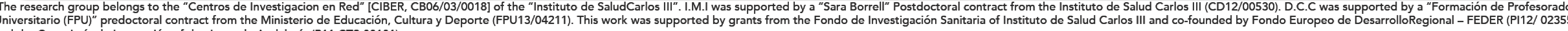

